\title{
A PROCEDURE FOR IMPROVING THE UPPER BOUND FOR THE NUMBER OF $n$-OMINOES
}

\author{
D. A. KLARNER AND R. L. RIVEST
}

1. Introduction. We begin with some definitions and a formulation of the problem treated in subsequent sections. Also included in this section is a brief indication of some of the known results dealing with the $n$-omino enumeration problem. Some of what follows together with more details may be found in $[3]$ or $[4]$.

Let $C$ denote the set of all integer points in the Cartesian plane, that is, $C=I \times I$ where $I$ denotes the set of all integers. Elements of $C$ are called cells, and two cells are said to be connected if the distance between them in the Cartesian plane is 1 . The set of cells $C$ may be regarded as the vertex set of an infinite planar graph $R$ whose edges consist of all pairs of connected cells in $C$. For each natural number $n$, let $R(n)$ denote the connected subgraphs of $R$ having exactly $n$ vertices. Clearly, $R(n)$ has infinitely many elements for each number $n$, but we are only interested in certain equivalence classes defined on $R(n)$ by means of the automorphism group $\mathscr{S}$ of $R$.

The automorphism group $\mathscr{S}$ of $R$ consists of isometries of the plane which map $C$ onto $C$; more precisely, an element of $\mathscr{S}$ is the restriction of such an isometry to $C$. An important subgroup $\mathscr{T}$ of $\mathscr{S}$ corresponds to the set of translations of the plane which map $C$ onto $C$. All of the elements of $\mathscr{S}$ may be formed by combining the elements $\mathscr{T}$ with combinations of some of the following isometries of the plane: reflection along the $x$-axis or $y$-axis, $90^{\circ}, 180^{\circ}$, or $270^{\circ}$ rotation about the origin.

Two elements of $R(n)$ are said to belong to the same translation class if one of these elements can be transformed into the other by an element of $\mathscr{T}$. The set of all translation classes induced in $R(n)$ by $\mathscr{T}$ is denoted by $T(n)$. Representative elements of the translation classes induced in $R(3)$ by $\mathscr{T}$ are shown in Figure 1. In the figure, boxes have been drawn around the cells of the animals, and the vertices and edges of the graphs have not been indicated in the conventional way.

Two elements of $R(n)$ are said to be the same if one of them can be transformed into the other by an element of $\mathscr{S}$. The set of equivalence classes induced in $R(n)$ by $\mathscr{S}$ is denoted $S(n)$. Representative elements of the equivalence classes induced in $R(3)$ by $\mathscr{S}$ are shaded in Figure 1. Read [6] called elements of $R(n)$ and $S(n)$ fixed and free animals respectively.

Received February 21, 1972 and in revised form, June 9, 1972. 

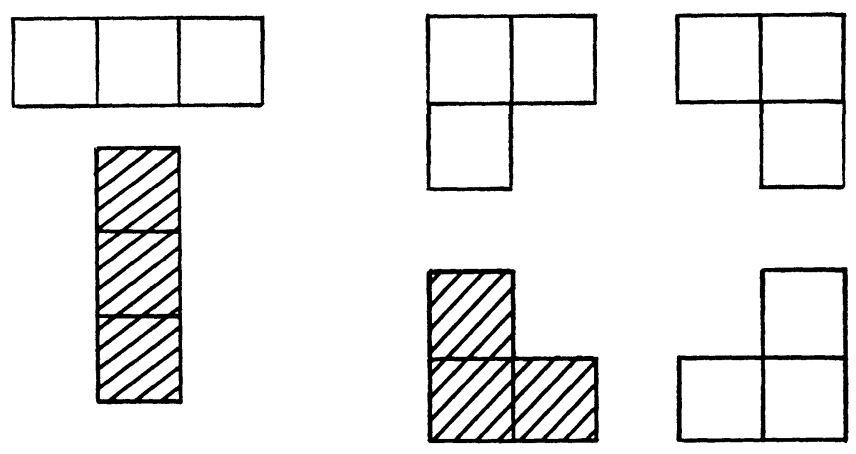

FIGURE 1. Representative elements of classes in $T(3)$

Let $t(n)=|T(n)|$ and $s(n)=|S(n)|$, then it follows from the definitions that

$$
\frac{1}{8} t(n) \leqq s(n) \leqq t(n) \leqq s s(n) \quad(n=1,2, \ldots) .
$$

Furthermore, it was shown in [3] that (i) the limits

$$
\theta=\lim _{n \rightarrow \infty}(t(n))^{1 / n}, \quad \theta^{\prime}=\lim _{n \rightarrow \infty}(s(n))^{1 / n}
$$

exist, (ii) $\theta^{\prime}=\theta$, (iii) $\theta \geqq(t(n))^{1 / n}$ for all $n$, and (iv) $\theta>3.72$. This last result was an improvement over $\theta>3.14$ and $\theta>3.20$ given in Eden [1], and [5] respectively. Also, Eden showed that

$$
t(n) \leqq\left(\begin{array}{c}
3 n \\
n-1
\end{array}\right)
$$

and since

$$
\lim _{n \rightarrow \infty}\left(\left(\begin{array}{c}
3 n \\
n-1
\end{array}\right)\right)^{1 / n}=\frac{27}{4},
$$

it follows from this result that $\theta \leqq 6.75$. Thus, the best bounds on $\theta$ after [3] were

$$
3.72<\theta \leqq 6.75 \text {. }
$$

Read [6] (for more details see also [4]) gave a method for computing the generating function for the number of elements of $T(n)$ involving $n$-ominoes whose cells occupy no more than $r$ rows of cells in the plane. For example, when $r=2$ this generating function is $\left(1+x^{2}\right) /\left(1-2 x+x^{4}\right)$. In general, Read's method gives rise to a rational function $p_{r}(x) / q_{r}(x)$ with $p_{r}$ and $q_{r}$ relatively prime polynomials such that $q_{r}(0)=1$. Thus, if the largest real root of $q_{r}(1 / x)$ is $\alpha_{r}$, then it follows that $\alpha_{i} \leqq \alpha_{\imath+1} \leqq \theta$ for $i=1,2, \ldots$. Therefore, this method leads to a procedure for improving lower bounds on $\theta$ indefinitely. Furthermore, Ashok Chandra, a graduate student in the Computer Science Department at Stanford University has shown us a proof that the sequence $\left(\alpha_{i}: i=1,2, \ldots\right)$ converges to $\theta$. It might be remarked that 
the amount of work required by this method to improve the bound $3.72<\theta$ (proved by an entirely different method in [3]) appears to be prohibitive.

An alternative procedure for improving lower bounds on $\theta$ indefinitely was proposed by the late Leo Moser. Consider the set $W(n)$ of translation classes of $n$-ominoes $X$ such that $X$ has exactly one cell in its bottom row and more than one cell in all other rows; also, one cell in the top row of $X$ is to be distinguished from the other cells. For example, $W(1)$ has one element, $W(2)$ is empty, and $W(3)$ has four elements. Figure 2 illustrates the elements of $W(4)$; the distinguished cells in top rows are marked with a cross. Now we use
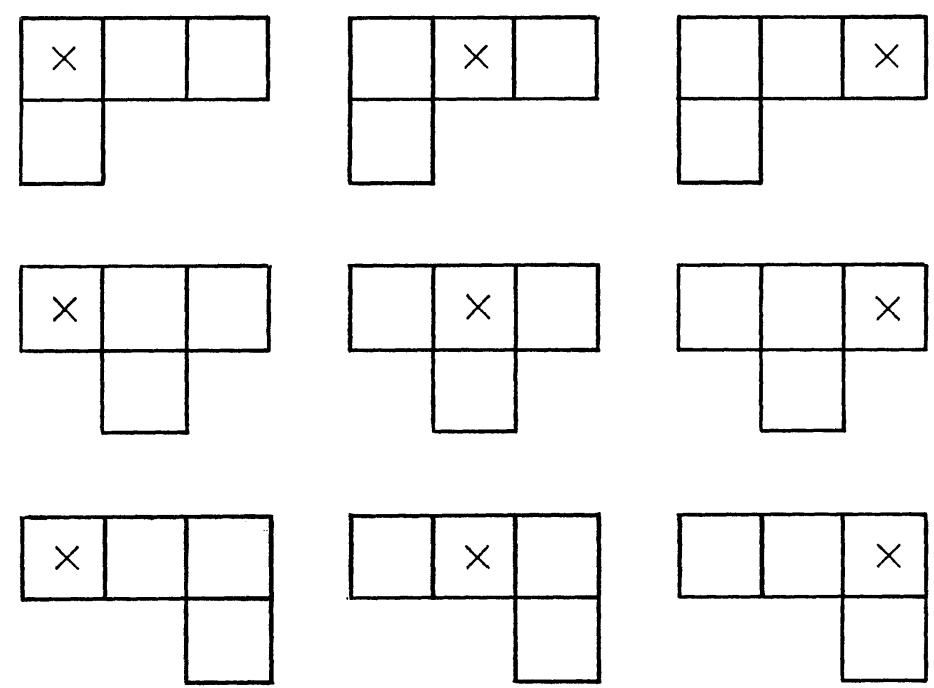

FIGURE 2. Elements of $W(4)$

elements of $W(1) \cup W(2) \cup \ldots$ to construct elements of a set $T^{*}(n)$ consisting of translation classes of $n$-ominoes $X$ such that $X$ has exactly one cell in its bottom row and a distinguished cell among the cells in its top row. Let $t^{*}(n)=\left|T^{*}(n)\right|$; then it is easy to see that

$$
t(n-1) \leqq t^{*}(n) \leqq n t(n)
$$

and this implies

$$
\lim _{n \rightarrow \infty}\left(t^{*}(n)\right)^{1 / n}=\theta .
$$

Now we estimate $t^{*}(n)$ from below. Every element $X \in T^{*}(n)$ corresponds to a unique sequence $\left(X_{1}, \ldots, X_{k}\right)$ with $X_{1} \in W\left(n_{1}\right), \ldots, X_{k} \in W\left(n_{k}\right)$ where $k, n_{1}, \ldots, n_{k}$ are certain numbers uniquely determined by $X$ with $n=n_{1}+\ldots+n_{k}$. This sequence is found by cutting $X$ into pieces with lines running along the bottom of each row of $X$ containing exactly one cell. 
The element $X_{i}$ lies between the $i$ th and $(i+1)$ st of these lines, and the distinguished cell in the top row of $X_{i}$ is either the distinguished cell of $X$ (in case $i=k$ ), or it is the cell joined to the unique cell in the bottom row of $X_{i+1}$. Letting $w(n)=|W(n)|$, it follows that

$$
t^{*}(n)=\sum w\left(n_{1}\right) \ldots w\left(n_{k}\right)
$$

where the sum extends over all compositions $\left(n_{1}, \ldots, n_{k}\right)$ of $n$ into $k$ positive parts for $k=1,2, \ldots, n$. If $\left(w^{*}(1), w^{*}(2), \ldots\right)$ is any sequence of nonnegative numbers such that $w^{*}(n) \leqq w(n)$, then of course

$$
t^{*}(n) \geqq \sum w^{*}\left(n_{1}\right) \ldots w^{*}\left(n_{k}\right) .
$$

Setting

$$
f(x)=\sum_{n=1}^{\infty} w^{*}(n) x^{n}
$$

we have

$$
\frac{f(x)}{1-f(x)}=\sum_{n=1}^{\infty}\left\{\sum w^{*}\left(n_{1}\right) \ldots w^{*}\left(n_{k}\right)\right\} x^{n}
$$

where the index of summation for the inner sum is the same as in (7). The coefficient of $x^{n}$ in the power series in (10) is a lower bound for $t^{*}(n)$ so long as $0 \leqq w^{*}(n) \leqq w(n)$. Thus, if we define

$$
f_{r}(x)=\sum_{n=1}^{r} w(n) x^{n}
$$

and define a sequence $\left(t_{r}^{*}(1), t_{r}^{*}(2), \ldots\right)$ by

$$
\frac{f_{r}(x)}{1-f_{r}(x)}=\sum_{n=1}^{\infty} t_{r}^{*}(n) x^{n}
$$

then it follows that

$$
t_{r-1}^{*}(n) \leqq t_{r}^{*}(n) \leqq t^{*}(n)
$$

for $r=1,2, \ldots$ and $n=1,2, \ldots$. Furthermore, if we put

$$
\varphi_{r}=\lim _{n \rightarrow \infty}\left(t_{r}^{*}(n)\right)^{1 / n},
$$

then $\varphi_{1} \leqq \varphi_{2} \leqq \ldots \leqq \theta$. Finally, we come to the computation of $\varphi_{r}$. Since $f_{r}(x) /\left(1-f_{r}(x)\right)$ is a rational function which generates a sequence of increasing positive integers, it follows that $\varphi_{r}$ is equal to the largest real root of the equation $f_{r}(1 / x)-1=0$. Thus, Moser's procedure comes down to enumerating the sets $W(1), \ldots, W(r)$ to find $\varphi_{r}$. One has more and more work to find improvements by this method, and indeed, so far no one has had the ambition required to calculate $\varphi_{r}$ for a large enough number $r$ to improve the bound $3.72<\theta$. 
So far we have seen two procedures for improving lower bounds on $\theta$ indefinitely. No such procedure is known for improving the upper bound on $\theta$, and it is our goal in this paper to show that such a procedure exists. Furthermore, we shall achieve a considerable improvement over Eden's bound $\theta \leqq 6.75$. The next section deals with combinatorial aspects of this problem which lead to a technical problem involving generating functions. This problem is dealt with in the third section, and in the final section we discuss the calculations which lead to our new upper bound for $\theta$.

2. $n$-ominoes viewed as sequences of twigs. In this section we develop an idea which originates with Eden [2]. We begin with a description of this idea, reformulating it so that our development appears straightforward. The idea is that a unique planted plane tree $E_{X}$ embedded in $R$ may be associated with each $n$-omino $X$. The tree $E_{X}$ is then interpreted as a sequence of "twigs", that is, certain small subtrees also embedded in $R$. Eden's set of twigs $E$ (shown in Figure 3) is finite, and each $Y \in E$ is assigned a weight $w(Y)=$ $x^{a} y^{b}$, where $a$ denotes the number of cells in $Y$ less 1 , and $b$ denotes the number of dead cells in Y. (Dead cells are coloured black in Figure 3: they will be explained later.) Let $E^{k}$ denote the set of all sequences of elements of $E$ having length $k$ for $k=0,1, \ldots$, and define the weight of $\bar{Y} \in E^{k}$ to be $W(\bar{Y})=x w\left(Y_{1}\right) \ldots w\left(Y_{k}\right)$ where $\bar{Y}=\left(Y_{1}, \ldots, Y_{k}\right)$ for $k=1,2, \ldots$, and define the weight of the empty sequence to be $x$.

It turns out that sequences of twigs corresponding to elements of $T(n)$ have weight $x^{n} y^{n}$, and the sum of the weights of all finite sequences of elements of $E$ is

$$
\begin{aligned}
x\left[1-\sum_{Y \in E} w(Y)\right]^{-1} & =\sum_{k=0}^{\infty} x\left[\sum_{Y \in E} w(Y)\right]^{k} \\
& =\sum_{k=0}^{\infty} \sum_{Y \in E^{k}} W(\bar{Y}) .
\end{aligned}
$$

Since $\sum w(Y)=y(1+x)^{3}$, the generating function given by (1) is

$$
\frac{x}{1-y(1+x)^{3}}=\sum_{n=0}^{\infty} x y^{n}(1+x)^{3 n}=\sum_{n=0}^{\infty} \sum_{m=1}^{3 n+1}\left(\begin{array}{c}
3 n \\
m-1
\end{array}\right) x^{m} y^{n} .
$$

Thus, if it is shown that there exists an injection of $T(n)$ into the set of finite sequences of $E$ having weight $x^{n} y^{n}$, then we are justified in concluding that the coefficient $e(n, n)$ of $x^{n} y^{n}$ in this power series is an upper bound for $t(n)$. Hence, if $1 / \epsilon$ is the radius of convergence of the "diagonal" power series of (2), that is, the power series

$$
\sum_{n=0}^{\infty} e(n, n) z^{n},
$$



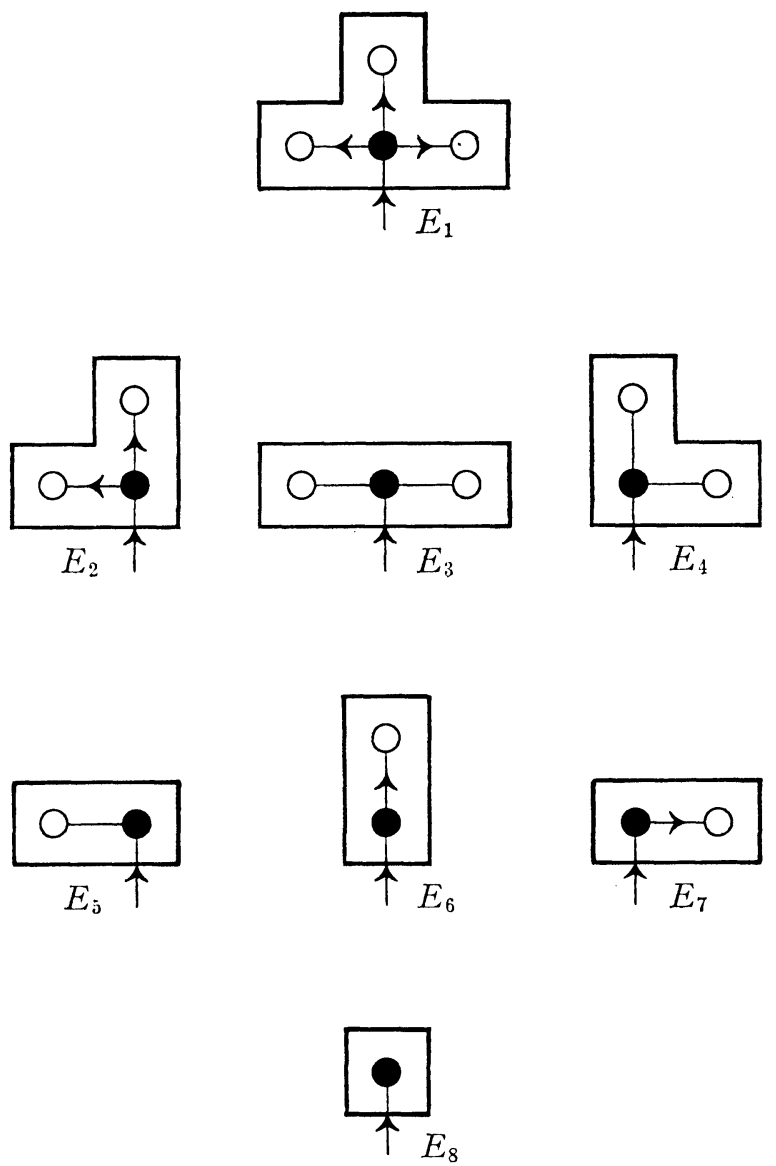

Figure 3. Eden's set of twigs $E$

then $\theta \leqq \epsilon$ where $\theta$ is defined in (1.2). But,

$$
e(n, n)=\left(\begin{array}{c}
3 n \\
n-1
\end{array}\right) \text { and } \epsilon=\frac{27}{4},
$$

and this is Eden's result mentioned in $\S 1$.

It remains to describe an injection of $T(n)$ into the set of finite sequences of elements of $E$ having weight $x^{n} y^{n}$. Suppose $X \in R(n)$; then a spanning tree $E_{X}$ of $X$ which is at the same time a planted plane tree embedded in $R$ may be defined as follows: Assign labels 1 and 0 to the left-most cell in the bottom row of $X$ and the cell below this one respectively, then draw an edge from cell 0 into cell 1 . Now we define a process which generates a spanning tree of $X$ assigning labels $1, \ldots, n$ to the vertices of $X$. See Figure 4 for an example of a spanning tree created by this process; the sequence of twigs in this example is $\left(E_{4}, E_{4}, E_{8}, E_{3}, E_{6}, E_{8}, E_{8}, E_{6}, E_{7}, E_{8}\right)$, and

$$
x w\left(E_{4}\right) \ldots w\left(E_{8}\right)=x \cdot x^{2} y \cdot x^{2} y \cdot y \cdot x^{2} y \cdot x y \cdot y \cdot y \cdot x y \cdot x y \cdot y=x^{10} y^{10} .
$$




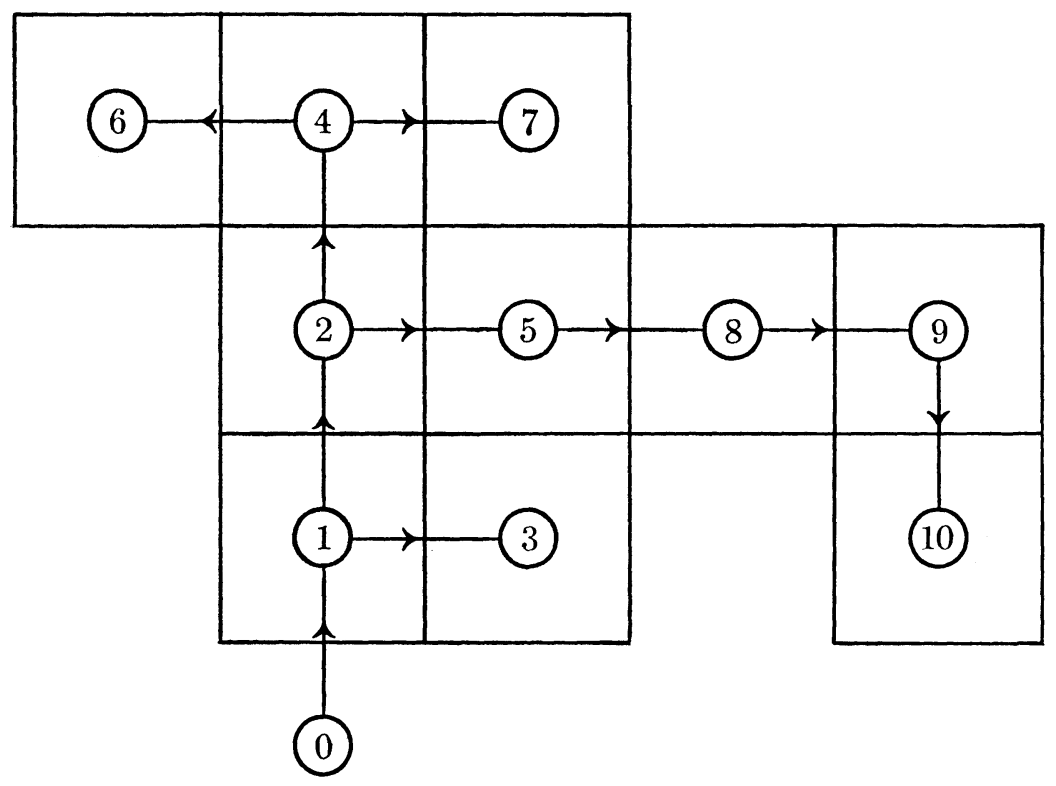

FIGURE 4. A spanning tree generated by Eden's method

The process consists of a sequence of $n$ steps $P(1), \ldots, P(n)$ of which the general step $P(i)$ is as follows: An edge has been drawn from cell $j_{i}$ i nto cell $i$. Three cells together with cell $j_{i}$ surround cell $i$ which for the moment we call $a_{i}, b_{i}, c_{i}$ going clockwise around cell $i$ from cell $j_{i}$. If $a_{i}$ is a cell of $X$ and has not been labelled earlier in this process, then an edge is drawn from cell $i$ into $a_{i}$, and $a_{i}$ is assigned the successor of the last label used in this process. Repeat this for $b_{i}$ and $c_{i}$, and go on to $P(i+1)$ or stop if $i=n$. It can be shown easily by induction that carrying out $P(1), \ldots, P(n)$ creates a spanning tree of $X$ which is also a planted plane tree embedded in $R$. At vertex $i$ in this tree we find exactly one of the twigs shown in Figure 3; denote this twig by $Y_{i}$, and define $E_{X}=\left(Y_{1}, \ldots, Y_{n}\right)$.

It is an easy matter to check that the weight of $E_{X}=\left(Y_{1}, \ldots, Y_{n}\right)$ is $x^{n} y^{n}$ for all $X \in R(n)$. Furthermore, if $X, X^{\prime} \in R(n)$ and $X$ is a translation of $X^{\prime}$, then $E_{X}=E_{X^{\prime}}$. Thus, the spanning tree of a representative element of a translation class of $n$-ominoes is representative of the spanning trees of all the $n$-ominoes in the translation class. This completes the description of an injection of $T(n)$ into the set of finite sequences of elements of $E$ having weight $x^{n} y^{n}$.

Our development of Eden's idea now follows naturally. The spanning tree $E_{X}$ of $X \in R(n)$ may be viewed as a sequence of elements selected from a set of "larger" twigs. For example, such a set of twigs may be defined for $k=1,2, \ldots$ as follows: Let $E(k)$ denote the set of all partial planted plane trees $Z$ embedded in $R$ such that (1) the dead cells of $Z$ are connected to the 
root of $Z$ with a path of length less than $k$, and (2) $Z$ must be a subtree of the partial spanning tree of some polyomino. The weight of an element $Y \in E(k)$ is defined to be $w_{k}(Y)=x^{a} y^{b}$ where $a$ denotes the total number of cells in $Y$ less 1 , and $b$ denotes the total number of dead cells in $Y$. The weight of a sequence $\bar{Y}=\left(Y_{1}, \ldots, Y_{r}\right)$ of elements of $E(k)$ is defined to be $W_{k}(\bar{Y})=$ $x w_{k}\left(Y_{1}\right) \ldots w_{k}\left(Y_{r}\right)$. Every $n$-omino $X$ gives rise to a unique sequence of elements of $E(k)$, and it can be shown by induction that the weight of such a sequence is $x^{n} y^{n}$. It follows from these definitions that $E(1)=E$, and the elements of $\mathrm{E}(1)$ are shown in Figure 3. The elements of $E(2)$ are compactly represented by the drawings in Figure 5 which are interpreted as follows: Each drawing represents the collection of twigs having in common the dead cells marked as black vertices. The elements of each collection are obtained by including all subsets of the cells marked with square vertices as white cells of a twig. The sum of the weights of all the twigs in each collection is written below each drawing.

Following (1), the sum of the weights of all finite sequences of elements of $E(k)$ is given by

$$
x\left[1-\sum_{Y \in E(k)} w_{k}(Y)\right]^{-1}=\sum_{m, n=0}^{\infty} e_{k}(m, n) x^{m} y^{n},
$$

and the coefficient $e_{k}(n, n)$ of $x^{n} y^{n}$ is an upper bound for $t(n)$. Furthermore, it can be shown that $e_{k+1}(n, n) \leqq e_{k}(n, n)$ for $k=1,2, \ldots$ and all $n$, in fact, for any fixed $k$, strict inequality must hold for all sufficiently large $n$. (Since our final result does not depend on these claims, we shall not bother to prove them.) Thus, if $1 / \epsilon_{k}$ denotes the radius of convergence of the diagonal power series of the power series given in (5), we have $\epsilon_{1} \geqq \epsilon_{2} \geqq \ldots \geqq \theta$, where $\theta$ is defined in (1.2). In the next section we show how to compute an upper bound for $\epsilon_{k}$; in fact, it follows from the results proved there that $\epsilon_{1}=6.75$, $\epsilon_{2} \leqq 5.50$, and $\epsilon_{3} \leqq 5.25$. The amount of work required by this procedure for $k=4$ or 5 say, may not be prohibitive, and the upper bound for $\theta$ might be further improved by this method. However, there is a set of twigs more efficient than the extension of Eden's set and it is the procedure associated with this set that we plan to push to the limits of our computing ambition.

There are eight $L$-shaped 4 -sets of cells near a given cell $u$ which we call $L$-contexts of $u$; rather than take space to define these 4 -sets precisely, we merely picture them in Figure 6. Using this concept, we describe the set of twigs $L$ shown in Figure 7. Each element of $L$ is composed of the following things: (i) a root cell along with a specified $L$-context of this cell, (ii) a set (possibly empty) of open cells which is linearly ordered, and (iii) each open cell is assigned one of its $L$-contexts. In Figure 7 we have marked the $L$ context of a twig's root cell with asterisks, the root cell itself is coloured black, the open cells are coloured white, and the $L$-context assigned to each open cell is indicated with an $L$. Certain cells are indicated with a large cross; these cells are called forbidden cells and they will never become a cell of the sub-polyo- 


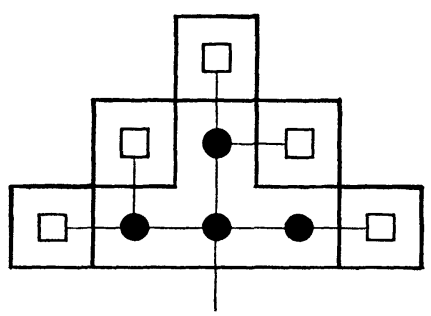

$$
x^{3} y^{4}(1+x)^{5}
$$

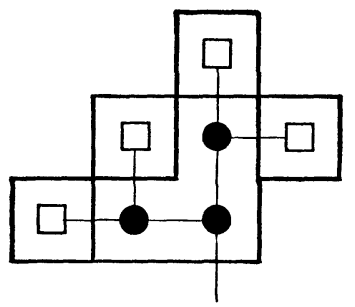

$x^{2} y^{3}(1+x)^{4}$

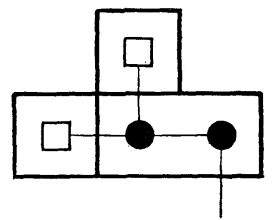

$x y^{2}(1+x)^{2}$

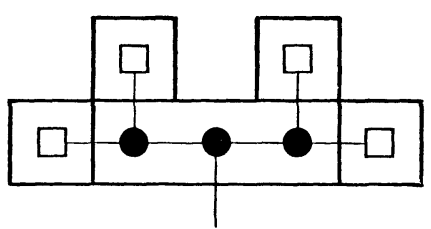

$x^{2} y^{3}(1+x)^{4}$

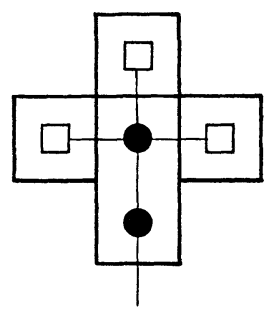

$x y^{2}(1+x)^{3}$

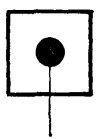

$y$

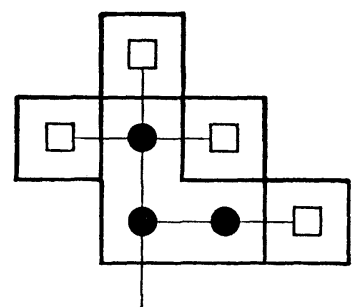

$x^{2} y^{3}(1+x)^{4}$

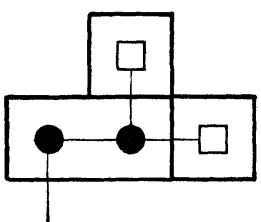

$x y^{2}(1+x)^{2}$

Figure 5. Elements of $E(2)$

mino of which the twig is a root. Where necessary (that is, in twigs $L_{3}$ and $L_{5}$ ), the linear order assigned to the open cells of a twig is indicated by numbering them.

Every element $X \in R(n)$ corresponds to a unique $n$-term sequence of elements of $L$. Just as in Eden's method, this sequence is constructed algorithmically by assigning a linear order to the cells of $X$ at the same time assigning an element of $L$ to each cell of $X$. The left-most cell $u$ in the bottom row of $X$ is cell 1 of $X$. The $L$-context $U$ of $u$ which consists of the cell to the left of $u$ and the three cells below $u$ form the $L$-context of a twig (which we will specify 

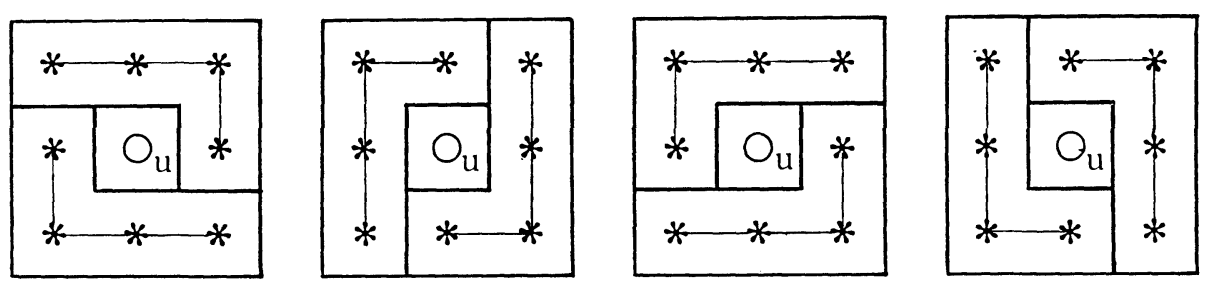

FIGURE 6. The eight $L$-contexts of cell $u$
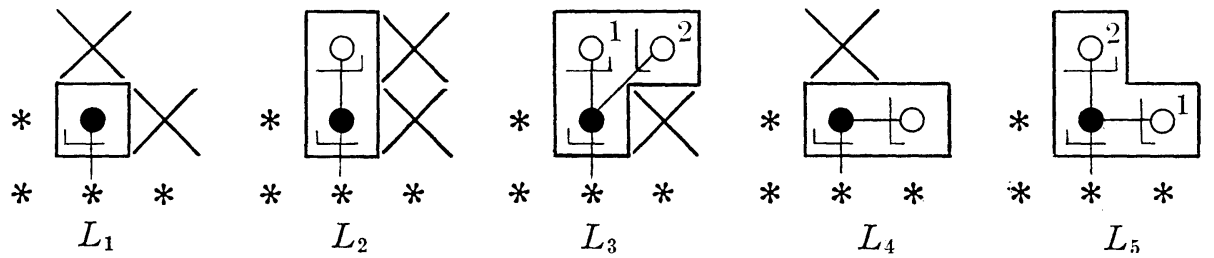

Figure 7. The set of twigs $L$

in a moment) whose root is $u$. Let $w$ and $y$ denote the cells connected to the right and above $u$, and let $x$ denote the cell $(\neq u)$ connected to $w$ and $y$. The twig assigned to $u$ (that is, cell 1 of $X$ ) is

(i) $L_{1}$, if $w, x, y \notin X$,

(ii) $L_{2}$, if $w, x \notin X, y \in X$,

(iii) $L_{3}$, if $w \notin X, x, y \in X$,

(iv) $L_{4}$, if $y \notin X$, $w \in X$,

(v) $L_{5}$, if $w, y \in X$.

It is easy to check that (i)-(v) cover all possible situations. The $L$-context of a twig is interpreted as a set of cells whose status of belonging or not belonging to $X$ is known. This is the case with the root of the twig assigned to $u$. Note however that this is true for cell 2 of twigs $L_{3}$ and $L_{5}$ only after the twig assigned to cell 1 has been specified. Now the linear order assigned to the cells of $X$ and the assignment of twigs to the cells of $X$ is carried out by doing $Q(2), \ldots, Q(n)$ where $Q(i)$ is defined as follows: Suppose labels $1, \ldots, j_{i-1}$ have been given to cells of $X$ with $j_{i-1}$ the last label given any cell. Go to cell $i$ of $X$ which is the open cell of a twig assigned to yet another cell of $X$, and let the $L$-context specified be the $L$-context of the twig to be assigned to cell $i$. All previously labelled cells of $X$ are deleted, and cell $i$ is viewed as the root cell of some connected component of $X$. Now the twig assigned to cell $i$ is determined in the same way as for cell 1 , and the open cell (or cells) belonging to this twig is (are) labelled $1+j_{i}$ (or $1+j_{i}$ and $2+j_{i}$ according to the linear order specified by the linear order of the open cells of the twig). Note that generally the $L$-context of cell $i$ may require one to reflect and/or rotate the appropriate twig to be assigned to it. The sequence of elements of $L$ assigned to $X$ by this method is defined to be $L_{X}=\left(X_{1}, \ldots, X_{n}\right)$ where $X_{i}$ 
is the twig assigned to cell $i$ by $Q(i)$. The spanning tree and sequence of twigs generated by this method corresponding to the decomino shown in Figure 4 is shown in Figure 8.

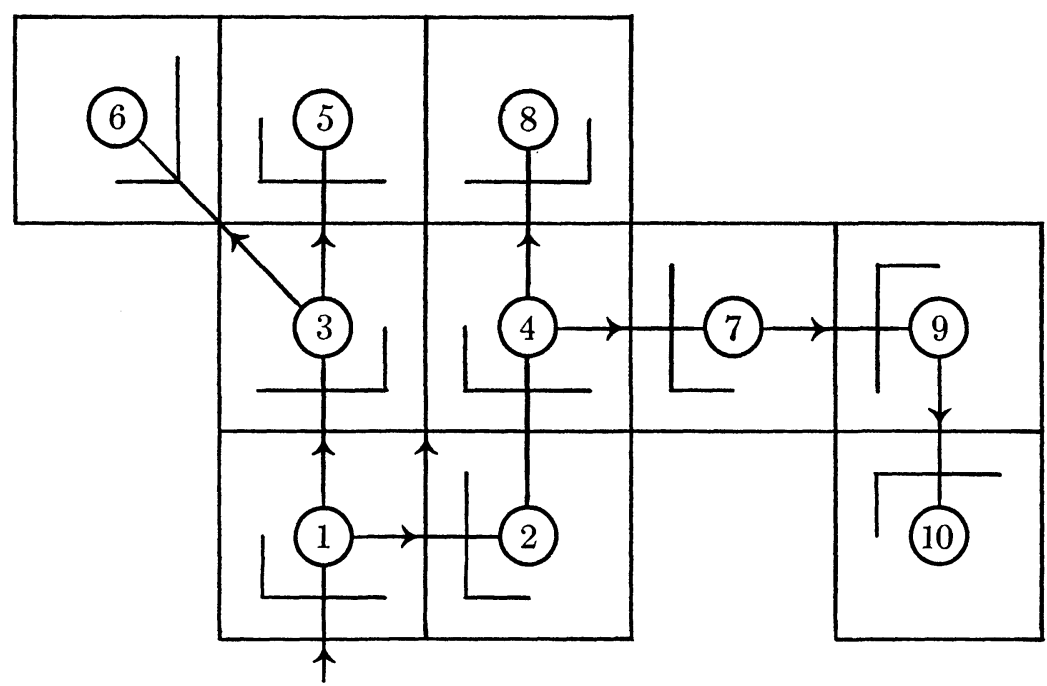

FIGURE 8. $\quad L_{X}=\left(L_{5}, L_{4}, L_{3}, L_{5}, L_{1}, L_{1}, L_{2}, L_{1}, L_{4}, L_{1}\right)$

Clearly, a common sequence is assigned to the elements of a translation class of $n$-ominoes, and $n$-ominoes belonging to different translation classes are assigned different sequences. Hence, there is an injection of $T(n)$ into the set of $n$-sequences of $L$. Furthermore, if the elements of $L$ are given the weights $w\left(L_{1}\right)=y, w\left(L_{2}\right)=x y, w\left(L_{3}\right)=x^{2} y, w\left(L_{4}\right)=x y, w\left(L_{5}\right)=x^{2} y$, and the weight of a sequence $\bar{X}=\left(X_{1}, X_{2}, \ldots\right)$ of elements of $L$ is defined to be $W(\bar{X})=x w\left(X_{1}\right) w\left(X_{2}\right) \ldots$, then $W\left(L_{X}\right)=x^{n} y^{n}$ for all $X \in R(n)$. Thus, there is an injection of $T(n)$ into the set of sequences of $L$ with weight $x^{n} y^{n}$.

Letting $l(m, n)$ denote the number of sequences of $L$ having weight $x^{m} y^{n}$, we can use (1) with $L$ in place of $E$ to find that

$$
\begin{aligned}
\sum_{m, n=0}^{\infty} l(m, n) x^{m} y^{n} & =\frac{x}{1-y\left(1+2 x+2 x^{2}\right)} \\
& =\sum_{n=0}^{\infty} x y^{n}\left(1+2 x+2 x^{2}\right)^{n} .
\end{aligned}
$$

Thus, $l(n, n)$ is equal to the coefficient of $x^{n-1}$ in $\left(1+2 x+2 x^{2}\right)^{n}$, that is,

$$
l(n, n)=\sum_{k=0}^{n}\left(\begin{array}{c}
n \\
k, k+1, n-2 k-1
\end{array}\right) 2^{n-k-1},
$$


but

$$
\begin{aligned}
\sum_{k=0}^{n}\left(\begin{array}{c}
n \\
k, k+1, n-2 k
\end{array}\right. & -1) 2^{n-k-1} \\
& =\frac{2^{n}}{\sqrt{ } 2} \sum_{k=0}^{n}\left(\begin{array}{c}
n, k+1, n-2 k-1
\end{array}\right)\left(\frac{1}{\sqrt{ } 2}\right)^{k}\left(\frac{1}{\sqrt{ } 2}\right)^{k+1} \\
& \leqq \frac{2^{n}}{\sqrt{ } 2}\left(\frac{1}{\sqrt{ } 2}+\frac{1}{\sqrt{ } 2}+1\right)^{n}<(2+2 \sqrt{ } 2)^{n}
\end{aligned}
$$

It follows that

$$
\theta=\lim _{n \rightarrow \infty}(t(n))^{1 / n} \leqq \lim _{n \rightarrow \infty}(l(n, n))^{1 / n} \leqq 2+2 \sqrt{ } 2<4.83,
$$

which is already a substantial improvement on Eden's bound $\theta \leqq 6.75$. We can improve further on $\theta<4.83$ using $L$ in analogy to our improvement on $\theta<6.75$ using $E$.

Consider the infinite set $H$ of twigs generated from the set of twigs $L$ in the following way. Every partial spanning tree of a polyomino is a member of $H$, where the spanning tree is generated with the procedure $Q$ corresponding to the set of twigs $L$. Each twig $X \in H$ is like a polyomino except that (i) it has a unique root cell indicated, (ii) a particular $L$-context is associated with the root cell, (iii) a spanning tree of $X$ is indicated, (iv) all nonterminal nodes of the spanning tree are dead cells, and ( $v$ ) some of the terminal nodes of the spanning tree may be open cells, each with an associated $L$-context. Thus, the subset of $H$ consisting of $n$-cell twigs with no open cells is isomorphic to $T(n)$.

A partial order $\leqq$ may be defined on $H$ as follows: For any $\bar{X}, \bar{Y} \in H$ put $\bar{X} \leqq \bar{Y}$ whenever

(i) $\bar{X}$ has fewer cells than $\bar{Y}$, and

(ii) the root of $\bar{X}$ has the same $L$-context as the root of $\bar{Y}$, and

(iii) the spanning tree of $\bar{X}$ is isomorphic to a subtree of $\bar{Y}$ rooted at the root of $\bar{Y}$.

In essence, $\bar{X} \leqq \bar{Y}$ whenever $\bar{Y}$ can be "grown" from $\bar{X}$ by repeatedly applying the process $Q$ to the open cells of $X$. The element $\emptyset$ (a twig of no cells) is considered to be the smallest element of $H$. The covering relation of $H$ ordered by $\leqq$ is a tree with root $\emptyset$. A finite subset $C(\neq\{\emptyset\})$ of $H$ is called a cut if every element of $H$ is comparable to some element of $C$ (for example, the set $L$ forms a cut of $H$ ). A cut is said to be minimal if no other cut is a proper subset of it.

Given a minimal cut $C$, it is easy to show that the spanning tree of any $n$-omino $\bar{X}$ can be uniquely decomposed into a spanning tree corresponding to elements of $C$. The set of twigs corresponding to $X$ are ordered by the label assigned to their root by the process $Q$. The set $C$ of twigs thus forms a "complete" set of building blocks, that is, a set of twigs capable of constructing any $n$-omino. Furthermore, using the weight function $w$ defined on $L$, we define the weight of an element $\bar{X}=\left(X_{1}, \ldots, X_{k}\right)$ of $C$ to be $w(\bar{X})=$ 
$w\left(X_{1}\right) \ldots w\left(X_{k}\right)$, and the weight of a sequence $\bar{Y}=\left(Y_{1}, \ldots, Y_{j}\right)$ of elements of $C$ is defined to be $W(\bar{Y})=x w\left(Y_{1}\right) \ldots w\left(Y_{j}\right)$. Thus, if $C_{X}$ denotes the sequence of elements of $C$ corresponding to $X \in R(n)$, then $W\left(L_{X}\right)=W\left(C_{X}\right)$. Hence, there is an injective mapping of $T(n)$ into the set of all sequences of $C$ having weight $x^{n} y^{n}$.

Next, suppose $C$ and $C^{\prime}$ are minimal cut sets, and every element of $C$ is less than or equal to some element of $C^{\prime}$; then we write $C \leqq C^{\prime}$. It is rather easy to prove that if $l_{C}(m, n)$ denotes the number of sequences of $C$ with weight $x^{m} y^{n}$, and if $C \leqq C^{\prime}$, then $l_{C}(n, n) \geqq l_{C^{\prime}}(n, n)$. The point is, if sequences of elements of $C$ and $C^{\prime}$ are converted into sequences of elements of $L$, then the sequences giving rise to the number $l_{C^{\prime}}(n, n)$ constitute a subset of the sequences giving rise to the number $l_{C}(n, n)$. Thus, for each sequence $\left(C_{1}, C_{2}, \ldots\right)$ of minimal cuts with $C_{1} \leqq C_{2} \leqq \ldots$, we have

$$
l_{C_{1}}(n, n) \geqq l_{C_{2}}(n, n) \geqq \ldots \geqq t(n) .
$$

To calculate $l_{C_{i}}(n, n)$ we use (1) with $C_{i}$ in place of $E$ :

$$
\frac{x}{1-\sum_{T \in C_{i}} w(T)}=\sum_{m, n=0}^{\infty} l_{C_{i}}(m, n) x^{m} y^{n} .
$$

Thus, estimating $l_{C i}(n, n)$ in (11) presents us with the problem of estimating from below the radius of convergence of the "diagonal function" of a rational double power series. We want to use the fact (implied by (10)) that if $1 / \lambda_{i}$ denotes the radius of convergence of $l_{C_{i}}(n, n) x^{n}$, then

$$
\lambda_{1} \geqq \lambda_{2} \geqq \ldots \geqq \theta \text {. }
$$

This is the problem treated in the next section.

3. The diagonal of a rational function. In this section we tell how to find a lower bound for the circle of convergence of the diagonal of a double power series which represents a rational function. More precisely, suppose $P(x, y)$ and $Q(x, y)$ are polynomials with integer coefficients such that $P(0,0)=1$ and $Q(x, y) / P(x, y)$ is in reduced form. Then consider the representation of $Q(x, y) / P(x, y)$ as a power series

$$
F(x, y)=\frac{Q(x, y)}{P(x, y)}=\sum_{m, n=0}^{\infty} f(m, n) x^{m} y^{n} .
$$

The diagonal of $F(x, y)$ is defined to be

$$
F_{D}(z)=\sum_{n=0}^{\infty} f(n, n) z^{n}
$$

In Section 2 we encountered the problem of determining an upper bound for

$$
\varphi=\liminf _{n \rightarrow \infty}(f(n, n))^{1 / n},
$$


that is, $\varphi^{-1}$ is a lower bound for the radius of convergence of $F_{D}(z)$. To solve this problem, we use the integral representation for $F_{D}(z)$ given in [2].

We can suppose there exist positive constants $\alpha$ and $\beta$ such that the power series in (1) represents $F(x, y)$ for all $x$ and $y$ such that $|x|<\alpha,|y|<\beta$. Thus, the function $F\left(s, z s^{-1}\right) s^{-1}$ regarded as a function of $s$ is represented by the Laurent power series

$$
F\left(s, z s^{-1}\right) s^{-1}=\sum_{m, n=0}^{\infty} f(m, n) z^{n} s^{m-n-1}
$$

inside the circular annulus

$$
A=\left\{s:|s|<\alpha,|z|\left|s^{-1}\right|<\beta\right\}=\left\{s:|z| \beta^{-1}<|s|<\alpha\right\}
$$

which is not empty provided $|z|<\alpha \beta$. Note that the residue of $F\left(s, z s^{-1}\right) s^{-1}$ at $s=0$ is just $F_{D}(z)$. Thus, if $\Gamma$ is a circle inside $A$ with its centre at $s=0$, then we can apply the residue theorem to conclude that

$$
F_{D}(z)=\frac{1}{2 \pi i} \int_{\mathrm{\Gamma}} F\left(s, z s^{-1}\right) s^{-1} d s ;
$$

furthermore, the integral on the right is the sum of the residues of $F\left(s, z s^{-1}\right) s^{-1}$ at the singularities enclosed by $\Gamma$. Now we take into account the special form of $F(x, y)$.

There exist numbers $u, v$ with $w=u+v$, together with polynomials $P_{0}(z), \ldots, P_{w}(z)$ with integer coefficients such that

$$
P(x, y)=\sum_{j=0}^{v} x^{-j} P_{v-j}(x y)+\sum_{j=1}^{u} x^{j} P_{v+j}(x y) .
$$

Using this form of $P(x, y)$, we have

$$
F\left(s, z s^{-1}\right) s^{-1}=\frac{s^{v-1} Q\left(s, z s^{-1}\right)}{\sum_{j=0}^{w} P_{j}(z) s^{j}} .
$$

There exist functions $\pi_{1}=\pi_{1}(z), \ldots, \pi_{w}=\pi_{w}(z)$ such that

$$
\sum_{j=0}^{w} P_{j}(z) s^{j}=P_{w}(z) \prod_{j=1}^{w}\left(s-\pi_{j}\right) .
$$

We shall assume the $\pi$ 's are distinct and treat this case only.

Suppose $\pi_{j}$ is inside $\Gamma$ for $j=1, \ldots, t$, and $\pi_{j}$ is outside $\Gamma$ for $j=t+1, \ldots, w$. Then we can combine (5), (7) and (8), and sum the residues of $F\left(s, z s^{-1}\right) s^{-1}$ at $\pi_{j}$ for $j=1, \ldots, t$ to find that

$$
F_{D}(z)=\sum_{j=1}^{t} \pi_{j}{ }^{v-1} Q\left(\pi_{j}, z \pi_{j}^{-1}\right)\left\{P_{w}(z) \prod_{k=1, k \neq j}^{w}\left(\pi_{j}-\pi_{k}\right)\right\}^{-1} .
$$

Hence, the singularities of $F_{D}(z)$ form a subset of the roots of the equation

$$
R(z)=P_{w}(z) \prod_{j, k=1 ; j \neq k}^{w}\left(\pi_{j}(z)-\pi_{k}(z)\right)=0,
$$


and a lower bound for the radius of convergence of $F_{D}(z)$ is the minimum modulus of all these roots. If it is known that $\{f(n, n): n=0,1, \ldots\}$ is an increasing sequence of integers, a lower bound for the radius of convergence of $F_{D}(z)$ is the smallest real root of $(10)$. Note that if the $\pi$ 's are not all distinct, then the product in $(10)$ is 0 , and this test fails.

Finally, $R(z)$, the function defined by the left member of $(10)$, is symmetric in $\pi_{1}, \ldots, \pi_{w}$, so $R(z)$ can be expressed as a polynomial in $P_{0} / P_{w}, \ldots, P_{w-1} / P_{w}$. In fact, $\pm R(z)$ is the discriminant of $s^{v} P\left(s, z s^{-1}\right) / P_{w}(z)$ regarded as a polynomial in $s$. This discriminant may be expressed as a determinant, and computed in terms of the quantities $P_{i} / P_{w}(i=0, \ldots, w-1)$ as in Uspensky [7, pp. 277-291]. We find

$$
\left(P_{w}(z)\right)^{2 w-2} R(z)=\operatorname{det} M(z),
$$

where $M$ is a $(2 w-1) \times(2 w-1)$ matrix whose first $w-1$ rows consist of cyclic shifts of $\left(P_{w}, P_{w-1}, \ldots, P_{0}, 0, \ldots, 0\right)$, and the next $w$ rows consist of cyclic shifts of $\left(w P_{w},(w-1) P_{w-1}, \ldots, P_{1}, 0, \ldots, 0\right)$.

Thus we are led to the following conclusion: If $F(x, y)$ is a rational function with the form given in (1), and if the diagonal $F_{D}(z)$ of $F(x, y)$ generates an increasing sequence of integers, then an upper bound for $\varphi$ defined in (3) is the largest real root of the polynomial equation

$$
\operatorname{det} M(1 / z)=0 .
$$

4. Computational results. The method outlined in the preceding sections was carried to the limits of practical computation to find an upper bound for $\theta$ (defined in (1.2)). In this section we describe in more detail the computational method used, and the results achieved.

A sequence of increasingly larger cut sets $C_{1}, C_{2}, \ldots$ of the set $H$ (as defined in $\S 2$ ) is precisely defined in this section. It will be clear that $C_{i}<C_{\imath+1}$ for $i=1,2, \ldots$, so that the radii

$$
\lambda_{1}{ }^{-1}, \lambda_{2}{ }^{-1}, \ldots \text { of } \sum l_{C_{1}}(n, n) x^{n}, \sum l_{C_{2}}(n, n) x^{n}, \ldots
$$

respectively decrease and are bounded below by $\theta$. Of course, this implies $\lim (i \rightarrow \infty) \lambda_{i}=\lambda$ exists, but we only know that $\lambda \geqq \theta$. We conjecture that $\lambda=\theta$.

The elements of $C_{i}$ were enumerated explicitly by a computer program written in SAIL (an ALGOL dialect) for the PDP-10 at the Artificial Intelligence Laboratory of Stanford University. The twigs of each cut set were displayed on a scope allowing us to verify the correctness of the program. The definition of $C_{i}$ and enumeration procedure are as follows.

The cut set $C_{i}$ is defined to be the set of all twigs having exactly $i$ dead cells together with the set of all twigs having fewer than $i$ dead cells and having no open cells. Every twig $T$ containing more than $i$ dead cells must contain 
a sub-twig rooted at the same cell as $T$ which has exactly $i$ dead cells. This demonstrates that $C_{i}<C_{j}$ for all $j>i$.

Some insight for our enumeration procedure may be gained from an understanding of the tree-structure of $H$ induced by the covering relation of the partial ordering defined on $H$. Recall the constituent parts of a twig: First, there is a root cell which is dead and marked to show the orientation of the twig in the plane. Apart from the root cell, the twig may have other dead cells. Also, the twig may have a set of open cells, and when they are present, these cells are assigned a definite linear order. Each of the open cells is marked to indicate the orientation of a twig which will eventually have its root superimposed on this cell. Finally, certain cells near the twig called forbidden cells are marked to indicate that no descendant of the twig includes any of these cells. The immediate descendants of a twig $T$ are constructed as follows: First, if $T$ has no open cells, then $T$ has no descendants. Next, if $T$ has any open cells, then there is a least open cell $X$, and $X$ has been marked to indicate the orientation of a twig to be joined to $T$ at $X$. Now we superimpose the root cell $Y_{i}$ of twig $L_{i}$ (shown in Figure 7 ) on $X$ for $i=1, \ldots, 5$ so that the orientation indicated on $Y_{i}$ matches the orientation on $X$. An element $L_{i} * T$ of $H$ is formed in this way if the following condition holds:

(*): None of the cells of $L_{i}$, except for $Y_{i}$, overlap any of the cells or forbidden cells of $T$, and none of the forbidden cells of $L_{\imath}$ overlap any cells of $T$.

If $L_{i} * T$ meets condition $(*)$, the construction is completed by including the forbidden cells of $L_{i}$ with those of $T$ to form the set of forbidden cells of $Y_{i} * T$. Next, cell $X$ is made a dead cell of $Y_{2} * T$. Note that this implies that every immediate descendant of $T$ (if any) has just one more dead cell than $T$. Finally, the linear ordering of the set of open cells of $T$ is modified in an obvious way to form a linear ordering of the open cells of $L_{i} * T$. The set of open cells of $L_{i} * T$ includes the set of open cells of $T$ with $X$ deleted together with the open cells (if any) contributed by $L_{i}$. The open cells of $L_{i}$ keep their relative order and become the largest elements in the new ordering.

Every twig $T$ may be assigned a unique sequence $\sigma(T)=\left(Z_{1}, \ldots, Z_{i}\right)$ having range $L=\left\{L_{1}, \ldots, L_{5}\right\}$. This sequence is selected so that $T=Z_{1} *\left(Z_{2} * \ldots\left(Z_{i}\right) \ldots\right)$. The fact that the sequence exists and is unique follows by induction. It follows from the observation that a twig's immediate descendants have one more dead cell, that the length $|\sigma(T)|$ of $\sigma(T)$ is the number of dead cells in $T$. Recall that the weight of a twig $T$ is defined to be $x w\left(Z_{1}\right) \ldots w\left(Z_{i}\right)$ where $\sigma(T)=\left(Z_{1}, \ldots, Z_{i}\right)$. If $T$ has no descendants and $i$ dead cells, it follows that $W(T)=x^{i} y^{i}$. Let

$$
H_{i}^{\prime}=\left\{T: T \in H, W(T)=x^{i} y^{i}\right\}
$$

and let

$$
H_{i}^{\prime \prime}=\left\{T: T \in H,|\sigma(T)|=i, W(T) \neq x^{i} y^{i}\right\} .
$$


Thus, now it follows from the definition of $C_{i}$ that

$$
C_{i}=H_{i}^{\prime \prime} \cup \bigcup_{j=1}^{i} H_{j}^{\prime}
$$

Our method involves the computation of

$$
W_{i}(x, y)=\sum_{T \in C_{i}} W(T)=W_{i}^{\prime \prime}(x, y)+\sum_{j=1}^{i} W_{i}^{\prime}(x, y)
$$

where

$$
W_{i}^{\prime \prime}(x, y)=\sum_{T \in H_{i}^{\prime \prime}} W(T), \quad W_{j}^{\prime}(x, y)=\sum_{T \in H_{j^{\prime}}} W(T) \quad(j=1,2, \ldots) .
$$

The following algorithm describes our procedure for computing $W_{i}(x, y)$ :

Step 1. Set $C_{\iota} \leftarrow \emptyset, B \leftarrow$ the set containing the single twig:

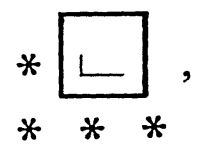

that is, a twig with one open cell and four context cells, and $W \leftarrow 0$.

Step 2. If $B=\emptyset$, stop $\left(C_{i}\right.$ is the desired cut set, and $\left.W=W_{i}(x, y)\right)$.

Step 3. Select any twig $T \in B$, and set $B \leftarrow B \backslash\{T\}$. If $T$ contains no open cells (that is, $T \in H_{j}^{\prime}$, for $j \leqq i$ ) or exactly $i$ dead cells (that is, $T \in H_{i}^{\prime \prime}$ ), set $C \leftarrow C \cup\{T\}, W \leftarrow W \cdot w(T)$, and return to Step 2 .

Step 4. For $i=1, \ldots, 5$, set $T_{i}=L_{i} * T$, and if $T_{i}$ meets condition (*) give above, set $B \leftarrow B \cup\left\{T_{i}\right\}$. Return to Step 2 .

This completes the description of the enumeration procedure. From the weight $W_{i}(x, y)$ thus calculated, the largest real roots of (3.12) can be found using a binary search method, using Gaussian elimination to evaluate $\operatorname{det} M(1 / z)$ at each value of $z$.

The results are summarized below. It should be noted that the largest real root of (3.12) for $i=10$ turned out to be 7.0075 . This root can be disregarded since $\lambda_{10}$ must be smaller than $\lambda_{9}$, so that the smallest root less than $\lambda_{9}$ is tabulated below. This phenomenon may be attributed to the fact that $(R(z))^{-1}$ has the same singularities as $F_{D}(z)$, but may have others besides these. From the table we conclude that

$$
\lim _{n \rightarrow \infty}(t(n))^{1 / n}=\theta \leqq 4.649551 .
$$




\section{Table 1}

\begin{tabular}{rrc}
$i$ & $\left|C_{i}\right|$ & $\lambda_{\boldsymbol{i}}$ \\
\hline \hline 1 & 5 & 4.828428 \\
2 & 21 & 4.828428 \\
3 & 93 & 4.828428 \\
4 & 409 & 4.796156 \\
5 & 1803 & 4.765534 \\
6 & 7929 & 4.738062 \\
7 & 34928 & 4.714292 \\
8 & 151897 & 4.690920 \\
9 & 656363 & 4.669409 \\
10 & 2821227 & 4.649551
\end{tabular}

\section{REFERENCES}

1. M. Eden, A two-dimensional growth process, Proceedings of the Fourth Berkeley Symposium on Mathematical Statistics and Probability, Vol. IV (Berkeley, California, 1961), pp. 223-239.

2. M. L. J. Hautus and D. A. Klarner, The diagonal of a double power series, Duke Math. J. 38 (1971), 229-235.

3. D. A. Klarner, Cell growth problems, Can. J. Math. 19 (1967), 851-863.

4. - Methods for the general cell growth problem, Combinatorial Theory and its Applications (Balatonfüred, Hungary, 1969), 705-720.

5. - Some results concerning polyominoes, Fibonacci Quart. 3 (1965), 9-20.

6. R. C. Read, Contributions to the cell growth problem, Can. J. Math. 14 (1962), 1-20.

7. J. V. Uspensky, Theory of equations (McGraw-Hill, New York, 1948).

Stanford University,

Stanford, California 